\title{
Survey on PV Modules' Common Faults After an O\&M Flight Extensive Campaign Over Different Plants in Italy
}

\author{
Francesco Grimaccia, Member, IEEE, Sonia Leva, Senior Member, IEEE, Alberto Dolara, Member, IEEE, \\ and Mohammadreza Aghaei, Student Member, IEEE
}

\begin{abstract}
Nowadays one of the most consolidated inspection methods for photovoltaic (PV) systems is the use of drones. PV modules installed in the last decade show now a quite wide range of defects able to sensibly compromise the performance of the plant. This paper aims to provide a quite comprehensive overview of typical defects observed after an extensive flight campaign in North of Italy made by light multicopter unmanned aerial vehicles over different types of PV plants. The objective of this investigation is to summarize the most observed detectable faults and to give some data of their frequency. Different kinds of sensors have been installed onboard and some peculiarities will be emphasized with respect to the visible, in-field measurements and IR-collected data. The results of this campaign will be discussed in order to provide a clear idea of potential impact of unmanned technology in this sector for future decades.
\end{abstract}

Index Terms-PV module faults, PV monitoring and control, unmanned aerial vehicle (UAV).

\section{INTRODUCTION}

$\mathbf{R}$ ENEWABLE energy sources contribution to power generation almost represents a valid alternative to limit fossil fuel usage and pollution for future decades. European countries have widely invested in alternative energies sources to reduce emissions and politically promote the so called clean energy. In Italy, during past years of generous public policies, the PV sector has witnessed a very rapid increase in its production rate with the complicity of low consumer prices and a general market euphoria.

However, the quality of the installed modules was not always guaranteed especially because produced in a rapid time frame, and often with characteristics below the common standards [1]. The race to install and meet such generous public incentives has left many solar installations across a general lacking in maintenance. After a relative short period, evident modifications in the visual aspect of some modules appeared and a quite large spectrum of defects started to be observed by operation and maintenance $(\mathrm{O} \& \mathrm{M})$ operators.

Manuscript received November 29, 2016; revised January 9, 2017; accepted February 22, 2017.

The authors are with the Department of Energy, Politecnico di Milano, Milano 20133, Italy (e-mail: francesco.grimaccia@ polimi.it; sonia.leva@ polimi.it; alberto.dolara@polimi.it; mohammadreza.aghaei@polimi.it).

Color versions of one or more of the figures in this paper are available online at http://ieeexplore.ieee.org.

Digital Object Identifier 10.1109/JPHOTOV.2017.2674977
TABLE I

MAIN FEATURES OF PV PLANTS UNDER ANALYSIS

\begin{tabular}{cccccc}
\hline \hline PV Plant & $P_{n}(\mathrm{~kW})$ & Year & Type of installation & Number of modules & Failure rate \\
\hline A & 997.92 & 2010 & Ground mounted & 4154 & $2.6 \%$ \\
B & 907.19 & 2011 & Rooftop mounted & 4010 & $81.8 \%$ \\
C & 997.60 & 2011 & Ground mounted & 4640 & $3.3 \%$ \\
D & 992.64 & 2012 & Ground mounted & 4224 & $<1 \%$ \\
E & 1634.85 & 2011 & Ground mounted & 7226 & $3.61 \%$ \\
\hline \hline
\end{tabular}

Some defects are generally due to the solar irradiation itself or temperature effects that can represent stress factor for PV module degradation, together with weather and particular environmental conditions (e.g., high ultraviolet components, temperature cycles, and fast chemical reactions [2]). In some harsh environment areas such as the deserts for example, sand, wind, and dust can also lead to dramatically decrease of PV modules performance [3]. In the last years, manufacturers have produced thin film solar cells and the thickness of absorber layers tended to be thinner with an increased solar cells area, making the cells more sensitive and fragile during shipment and installation [4]. Besides, early failures can turn into the complete damage of the PV modules: recently some smart methods aiming at detecting this degradation mechanism by means of indicators based on resistance have been proposed [5].

In this context, the authors started to investigate unmanned technology potential in the photovoltaics plant monitoring operations with an extensive flight campaign (see Table I). Many PV plants are too large and too time consuming to be inspected by manned technologies, especially if they are located on the roof of buildings or hard accessible areas. Thus, in recent research the introduction of unmanned aerial vehicle (UAV) technology in PV O\&M operations has been proposed to monitor and inspect different power plants. For this purpose, some ad hoc sensors have been tested and chosen as appropriate monitoring tools to scan PV modules in the most effective way. Previous results have shown that it is possible to have a good correlation between captured data and module healthy status when the flight procedures are well designed and planned [6].

As better explained in the follows, few attempts have been done in using unmanned technologies for PV system monitoring, mainly for sporadic experiments and small plants, and poor results have been published in the literature. Here, the procedure of utilizing UAV was successfully tested both for "ground" 
and "on roof" PV plants, with a systematic approach and some technical advantages for rooftop plants.

Thus, the main purpose of this paper is to summarize main results after a real extensive flight campaign performed in Italy over a different series of PV plants, both on roof and on ground. The original scope of this study was to introduce and test the unmanned technology itself with a cost effective approach, but in the end a large database of data, in visible and infrared spectrum, was available and relevant results could be inferred on different defects. In this path, the light weight UAV was transformed and adapted in order to conceive an ad-hoc machine optimized for the photovoltaic field arrays inspection and for computational postprocessing operations [6].

This paper is organized as follows. Section II introduces and summarizes peculiarities of UAV inspection method for PV faulty modules, giving details about the used methodology and complete system description. Section III reports common defects found in the different plants showing picture data captured by the UAV and correlation between visible and IR sensors. Section IV gives some indications with respect to observed typical defects, relative failure rates, then comparing our findings with relevant results in recent literature and finally drawing main conclusion in Section V.

\section{UAV-BASED PV MONITORING SYSTEMS}

In order to reduce the O\&M costs, early fault diagnosis plays a crucial role by enabling the long effective life of PV arrays. A recent work on low-cost and fast diagnosis method with optimized sensor locations to detect faulty strings has been carried out [7]. Unmanned technologies have many advantages in comparison with manned aircrafts especially in terms of safety, weight, and cost [8].

Traditionally many different methods have been used for solar plant inspection and monitoring. Visual inspection is still used for cracks, corrosion, snail trails, discoloration, and similar phenomena [9], [10]. The IR methods are able to extend the detectable range of defects to resistive soldering, shunting paths, bypass diodes, disconnections, etc. [11].

These methods are often time consuming, not practical for PV plants integrated into roof of buildings and sometimes it is difficult to evaluate impact of defect on the power output performance. Furthermore, not all the defects' origin can always be recognized and distinguished from one another.

On the other hand, laboratory tests are not feasible in terms of economic impact on the O\&M budget, often not cost effective and reasonable for large PV array fields, unless particular purposes are necessary. Thus, for example, accelerated tests and $I-V$ curves are not practical during normal plant operation, even if portable analyzer is available in the field for the maintenance team. The same is valid for other methods such as electroluminescence and ultrasonic inspection.

Based on the aforementioned market condition, it is useful to have a fast and reliable tool to detect and classify many defects at once. In this investigation, a reliable and cost effective method is proposed to monitor a large portfolio of PV plants by using a series of optimized platforms (see Fig. 1) conceived for multiple plants on a vast operating area. The proposed method aims to reduce the monitoring cost much more than other previous systems.

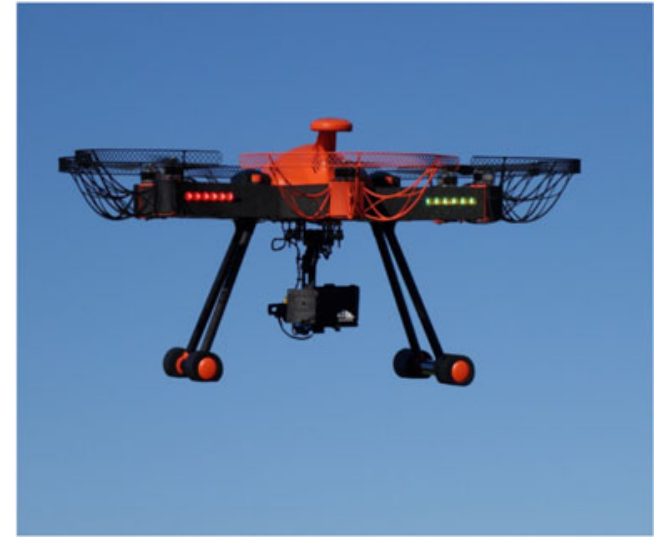

Fig. 1. PPL612-PV UAV model designed ad hoc for the extensive PV plant flight campaign.

Photovoltaic plants monitoring by unmanned aerial system has proved to offer many advantages in operations: fast detection, low cost, large area coverage, precise imagery, high flexibility, the ability to easily operate on roofs and hostile environment, and the integration with automatic systems. The last decade progress in the unmanned aerial industry technology, alongside improvements in sensor technology and control systems, has contributed to open up the massive use of UAVs in monitoring operation in different fields of civil application.

However, the technological factor is not the only issue to be taken into account since the regulatory framework is essential to properly operate with such systems, even in the energy field. The airworthiness context, for example, is still dynamic in different countries and in Italy, during these three last years where we performed our flight campaign, the civil aviation authority has published different and updated rules for remote-piloted aircrafts. In particular, the new Italian UAV regulation was recently issued by ENAC, essentially an update of its first edition (2013) on three categories' drones under $150 \mathrm{~kg}$, due to the dramatic increase in use and demands by the national industry and operators since that time [12]. An important amendment has been made with regard to permits for pilots: in order to operate UAV under $25 \mathrm{~kg}$ in visual line of sight conditions a remote pilot certificate is required.

The choice of different UAV properties is essential in order to make the most appropriate platform and relative sensors in the specific field of application. First attempt to use aerial solar thermography for integrated photovoltaic systems inspection was performed in Portland in 2012 but using a manned aircraft. In this experiment solar thermography was applied to determine the operational status of PV on a large scale [13]. Infrared thermography has the ability to see the heat profile among solar cells and can be used to determine damaged or defective modules, but other information can be added and correlated using multiple sensors mounted on the unmanned platforms [14].

Early failure detection plays a crucial role in PV plant maintenance to assure adequate performance, lifetime, and preventing defects' extension to healthy areas. Different aging mechanisms can also affect photovoltaic modules with correlated mismatching phenomena [15]. Among the vast range of possible modules failures known in the literature, namely cracked cells, yellowing, discoloration, bubbles, stratification, oxidation, corrosion, 
TABLE II

FEATURES OF PLP612-PV

\begin{tabular}{lc}
\hline \hline Items & Features \\
\hline Cruise speed range (PV) & $0-5 \mathrm{~m} / \mathrm{s}$ \\
Operational range (Regulation) & $1000 \mathrm{~m}(500)$ \\
Operational altitude (Regulation) & $5-50 \mathrm{~m}(150)$ \\
Flight endurance & $0.5 \mathrm{~h}$ \\
Weight & $5.3 \mathrm{~kg}$ \\
Height & $0.35 \mathrm{~m}$ \\
Length & $(\varnothing) 1 \mathrm{~m}$ \\
Propulsion & Electric Power $(6 \times 300 \mathrm{~W})$ \\
Operation wind speed (gust) & $5(10) \mathrm{m} / \mathrm{s}$ \\
Operation weather condition & Light rain \\
\hline \hline
\end{tabular}

delamination, microcracks, burnt cells, general hot spot, coating problems, foil detachment, improper framing, shading, and snail trails, we wanted to focus on the most relevant ones, better characterizing some of them. For example, a general hot spot can be associated with multiple causes based on shape and correlated information in the visible (see Section III). Thus, during the experimental campaign, the researchers have given many feedbacks to the UAV company operators in order to design and conceive an unmanned aircraft devoted to the photovoltaic field inspection. In this flight campaign, the ad hoc platform used in the different flights was a reliable and cost efficient proposed by the Nimbus company, the adapted model PLP612-PV (see Fig. 1), an optimized hexa-copter powered by electric energy. The main new features and design properties of Nimbus PPL612-PV platform are reported in Table II. The uniqueness of this UAV machine is the ad hoc design choices made from the outset to perform missions such as monitoring large energy plants: an iterative process during the PV flight campaign has allowed to optimize the copter features with respect to other multipurpose drones appeared in the market in the last years.

The novelty of this semi-automated system is its complete integration of heterogeneous data of the plant and the experimental validation on a large scale if compared with other sporadic test with UAVs traceable in the literature. The developed software is able to select, among the IR data, the images with potential defective modules. These files are then matched with the corresponding visual images in order to help the operator to identify and classify the specific failure. The system can also perform mosaicking and support the O\&M operator in the correct identification of the module placement in the plant map.

A first attempt with a similar approach for large plants has been recently proposed with a light UAV in France, but tested just for a $50 \mathrm{~kW}$ plant [16]. This experiment in fact was a first single small-scale PV plant installed on the rooftop of a building and different electrical mismatches were recognized under real field conditions with serious thermal patterns defined for $\Delta T>$ $20{ }^{\circ} \mathrm{C}$. In our experiment instead the system was tested on five different PV plants with relative features reported in Table I.

The proposed method depicted in Fig. 2 combines at the same time IR and visual images captured by the aerial perspective with direct measurements performed in the plant by using ad hoc instruments or the collected data by the on-site monitoring system if available. With a semi-automatic system, the georeferenced panel pictures are reported in a failure map which fully describe the plant status and in the same cases (see Fig. 8 and Section IV)

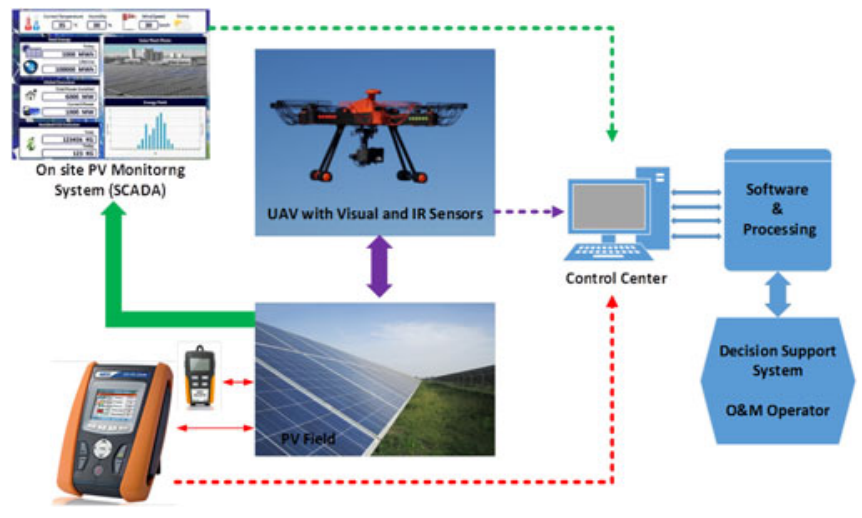

Fig. 2. Schematic of the automated control monitoring system for PV power plants based on UAV.

it is possible to correlate performance indexes with parts of the overall plant.

\section{Typical Defects And Main Experimental Results}

The extensive flight campaign using the UAV-based system shown in Fig. 2 has been applied on five different PV plants.

In this experimental work, two different synchronized cameras, namely a Panasonic Lumix GM1 HD $(4592 \times 3448)$ and FLIR A35 $(640 \times 512)$ thermal imager, are mounted on a light multirotor UAV machine to capture the pictures and sensor data of PV panels defects. In some of the accessible plant, an in-field measure with a grid analyzer (e.g., HT SOLAR 300N) was performed in order to compare energy performance with the failure maps and to the onsite monitoring system supervisory control and data acquisition (SCADA).

One of the main purpose of a survey of this type is to classify the PV module defects to find out early solution and prioritize main interventions. The interaction between measurements and UAV data allowed to reach two main results: to be able to better classify the genesis of defects and on the other side to estimate the severity of failures in terms of energy performance. Again understanding which kind of defect/failure can be detected by visual inspection, IR, electric measurements, or a proper combination of them is a very important task to design a cost-effective monitoring system.

According to our data collected in this extensive flight campaign, concerning just the PV modules, some considerations can be drawn in the follows.

A very common occurrence is when $1 / 3$ or $2 / 3$ of a module is hotter than the other parts (see Fig. 3). Since all the modules here analyzed are Si-crystalline with three by-pass diodes, this problem can be due to by-pass diode failure or to soldering defects in the junction box. In any case, we always classify this failure as by-pass failure. Even modules or string disconnections (see Fig. 4) are quite popular and they cause loss in efficiency of PV plant. They represent one of the three most common defects among the observed plant in Italy and they can be easily recognized just using IR sensors mounted onboard.

However, it is important to notice that not every defect has a direct impact on the output performance in power production, but often it is possible to find a direct correlation between observed faults and power degradation over time. 


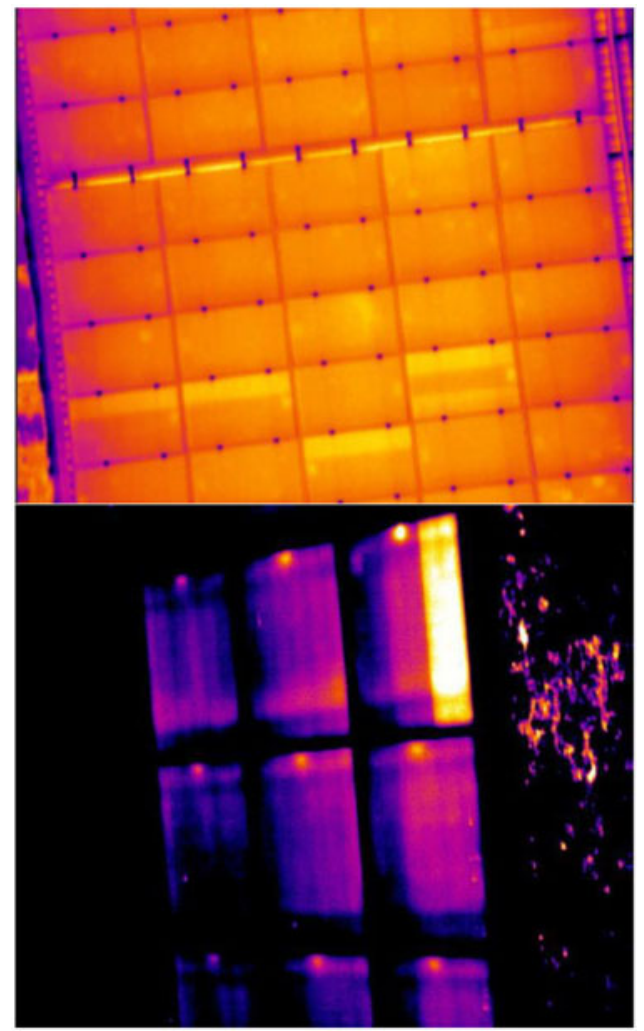

Fig. 3. Multiple (up) and single (down) by-pass failures observed with IR sensor in plant $\mathrm{B}$ and plant $\mathrm{D}$.

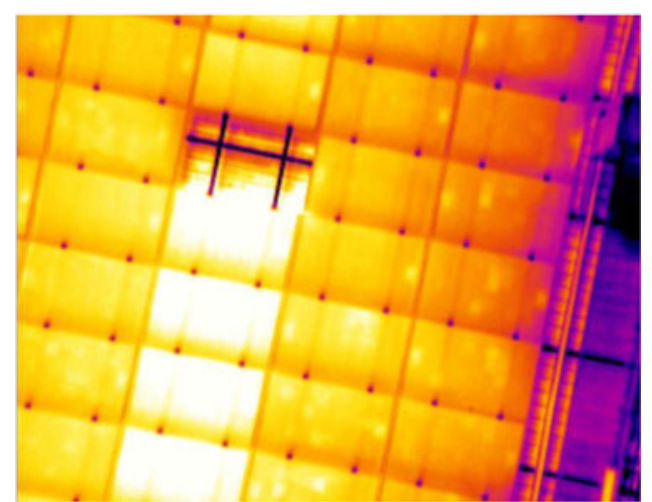

Fig. 4. Complete disconnected string with an empty module observed by the IR sensor in plant $B$.

Most visible failures appear on the PV modules due to different causes and this information is important for data correlation with IR pictures. In fact, if we focus for example on a general hotspot, it is not sufficient to fully characterize a particular defect without the information coming from the visible camera. Hot spot failures which are generally defined as an area on the PV module with higher temperature typically are associated with multiple reasons including cell mismatch, corrosion (see Fig. 5), dirty (see Fig. 6), or even partial shading (see Fig. 7). In this context, these kinds of failures (see Figs. 5 and 6) can be detected and classified only by combining visible and thermal cameras as in this field experiments since the IR only is not sufficient.

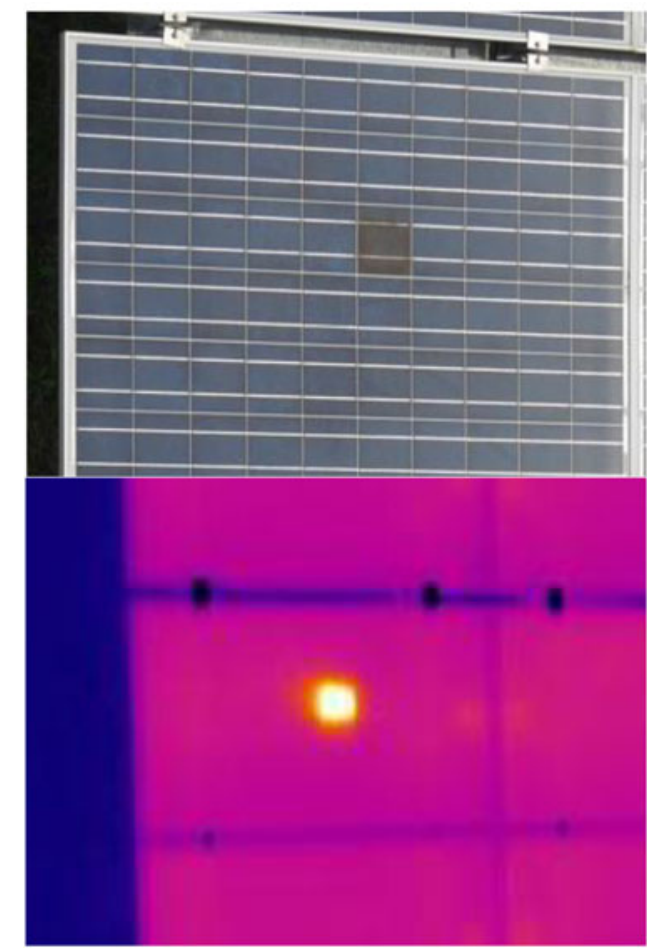

Fig. 5. Cell corrosion detected both in visible and IR in plant C.

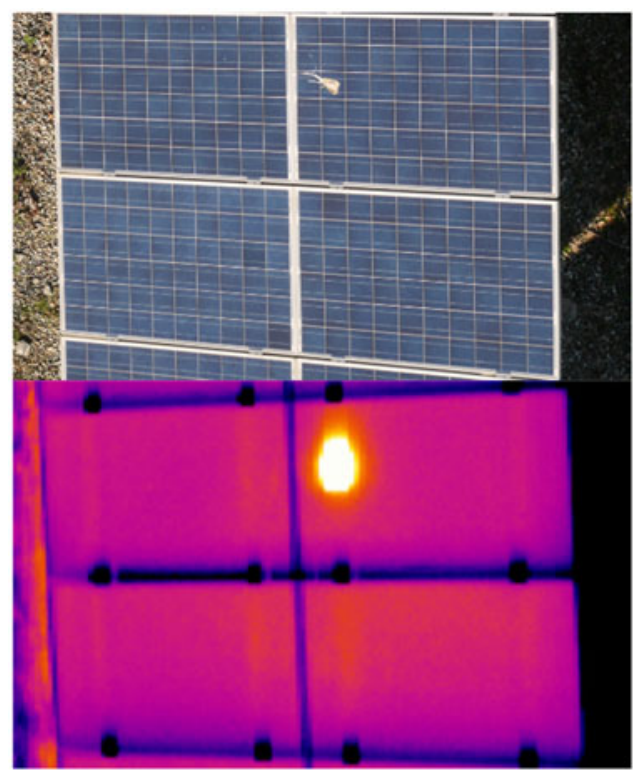

Fig. 6. Dirty module detection both in visible and IR in plant A.

Snail trail phenomenon, due to production causes linked to a particular manufacturer, appears as dark and irregular small lines (see Fig. 8). It is not so common according to our inspections, even if plant B was dramatically affected by this failure (92.2\% over only the found defected modules) while in plant E (12.5\% over only the found defected modules). It is important to notice that once again the correlation with visual pictures has made possible to classify as severe the snail trail in plant B while as incipient (with no thermal effect) in the case of plant E. Snail trail can be detected by visual inspection: in this case we 


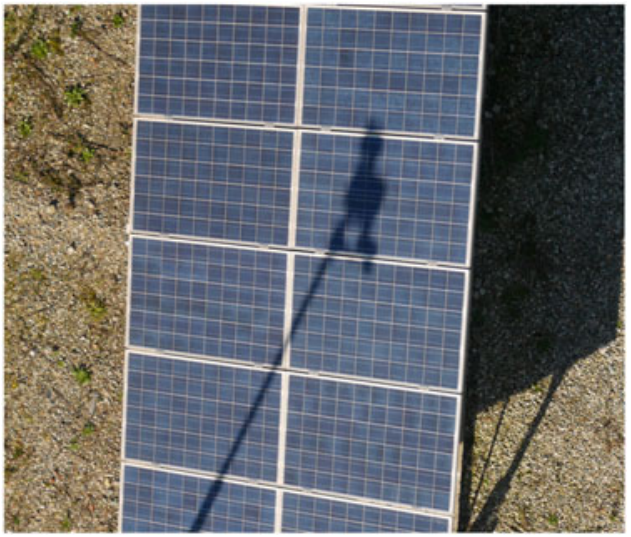

Fig. 7. Shading detection by a visual sensor in plant A.

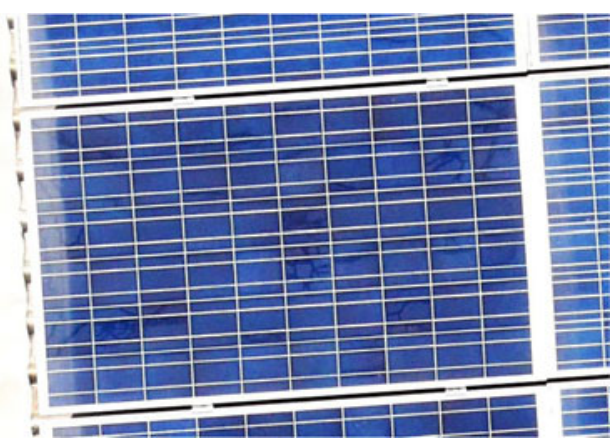

Fig. 8. Snail trail detection by a visual sensor in plant B.

need high-resolution sensors or to flight as close as possible to the module [17]. In some cases, snail trails are associated with cracks and then to hot spots [18].

Moreover, snail trails, shading, dirty, cracks which are not so hard to find according to this flight experience and can have a relevant influence on the PV modules performance can be all associated with IR hot spot phenomena and again the UAV correlation between the multiple sensors can give a complete picture of the observed defect.

As described in Section II the proposed method depicted in Fig. 2 combines IR and visual images with direct measurements performed in the plant. In fact, the combination of real-time measurement and UAV inspection provides important correlations between defects and output performance. For example, Fig. 9 shows the comparison between data recorded by the SCADA system and in-field measurements executed for the case of plant $\mathrm{D}$. In this case, the energy produced by the PV field is converted by using two separate inverters; thus, subsystem analysis is feasible in this case with disaggregated data.

The in-field measurements are then performed considering two different intervals corresponding to the measures made on inverters 1 and 2.

By the analysis of Fig. 9, it is possible to notice the incorrect calibration of the temperature plant sensor ( $\left.T_{c} S C A D A\right)$. Instead there is a good match between irradiance and power data recorded by SCADA and that measured in field (Meas.). Furthermore, a very strong correlation has been found between the final modules' defect map and the performance measured in each of the two separate parts of the plant with relative computed
TABLE III

Plant D Defect DetaIls Divided By INVERTERs 1 AND 2

\begin{tabular}{lcc}
\hline \hline Items & Inverter 1 & Inverter 2 \\
\hline$P R$ & $82.7 \%$ & $80.0 \%$ \\
Number of modules & 2112 & 2112 \\
Relevant defects & 6 & 25 \\
Other/minor defects & 110 & 118 \\
Failure rate & $0.3 \%$ & $1.2 \%$ \\
\hline
\end{tabular}

TABLE IV

FAILURE RATES OF OBSERVED DAMAgED Modules

\begin{tabular}{lc}
\hline \hline Defect - Failure & $\%$ Over only defected $(24254$ total inspected modules $)$ \\
\hline Discoloration/browning & $22.8 \%$ \\
Cracks in the cells & $<1 \%$ \\
Oxidation/corrosion & $<1 \%$ \\
Hot spot & $12.05 \%$ \\
Shading & $2.65 \%$ \\
Snail trails & $20.73 \%$ \\
Dirty & $3.24 \%$ \\
By-pass/disconnect. & $10.92 \%$ \\
Other/minor & $26.72 \%$ \\
\end{tabular}

performance ratios (PRs), see Table III. The most defected part in plant $\mathrm{D}$ in fact showed a $2 \%$ difference in PR calculated in the UAV flight time reference.

\section{ANALYSIS AND ReSUlts Discussion}

After our extensive flight campaign, it is possible to classify typical modules' defects and failures. The carried investigation with UAV platform was very important to identify both in visible and IR a fault catalogue with some first data about failures occurrences. Of course, the number of flights is still relatively small (under ten) but the overall number of modules inspected is now not negligible and some consideration can be done. Table IV reports the failure rates of most common observed defects, over the detected failures, in the five heterogeneous plants inspected by the UAVs. It is important to notice that reported values are indicative since the statistical basis after this first flight campaign is not yet sufficient to draw a robust and reliable picture on failure rates. If we consider [15] that among all the failures modes, inverter counts with a leading position for more than $40 \%$, ac subsystems around 15\%, and among others PV cells just for $2-3 \%$, the numbers reported in this study should be weighted accordingly in the O\&M perspective. Moreover, as anticipated, if we look for example at the snail trails phenomenon, in this picture its failure rate is significantly overestimated since one of the inspected plants was for three quarters affected by such a failure. The inspected plants are then not homogeneous, PV cell technology and plant structures differ.

The status of the observed plants varied from a percentage of defected panels less than 1-2\% which describes a good quality plant, to rate between 3 and $5 \%$ that represents a tolerable status, to a totally unacceptable one with more than $80 \%$ of faulty modules. However, some indications can be observed from this flight campaign and, apart from the peculiar case of the snail trails, hot spots and by-pass diodes show the highest rate of failures among the defected panels, followed by browning and dirty problems. It is important to notice that the category hot 

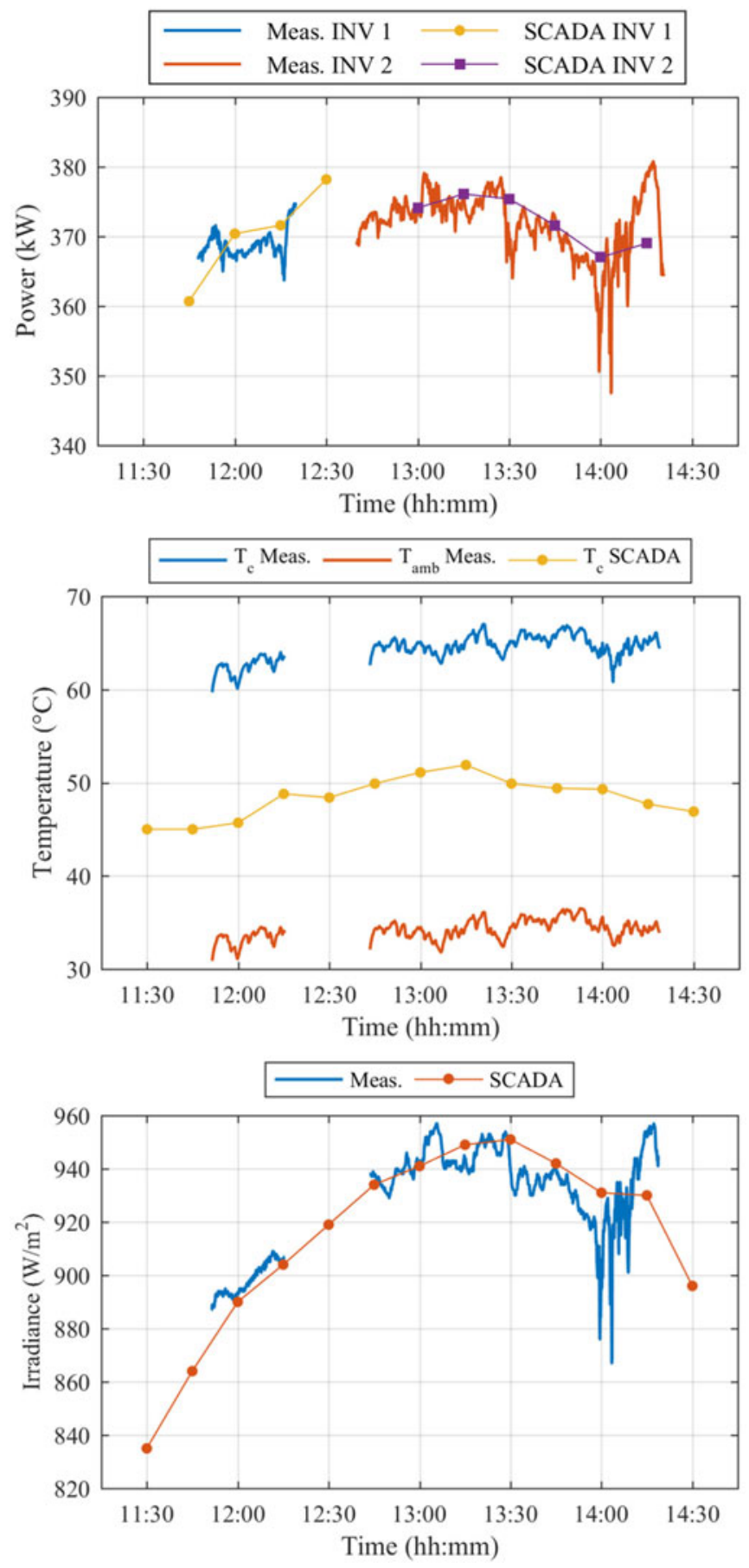

Fig. 9. Temperature, irradiance, and power comparison between data recorded by SCADA and measurement (Meas.) in plant D.

spot does not always identify a specific defect but often it is related or caused by other failures listed in Table III.

Early fault diagnosis represents a key factor in O\&M activity, but if we imagine to repeat more flights per year on the same plant the cost of the system becomes an issue. However, for large plants, especially on roof plants, the UAV system represents a real effective alternative. A complete manned IR inspection of $1 \mathrm{MW}$ plant may require 1-2 days, but with a comparable cost it is possible to fully inspect the same plant in just $1-2 \mathrm{~h}$, having

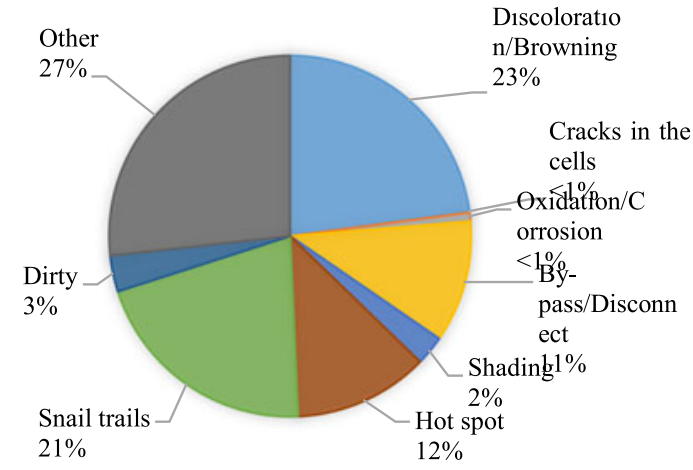

Fig. 10. Circle chart related to the module defects in the 5 plants (over the total number of failures).

a more complete information and a complete picture of the defects' origin thanks to the multiple sensors use.

With respect to the percentage of failures at module level found in this flight campaign (see Fig. 10), it is quite valuable comparing our findings with the recent literature even if not trivial and made on heterogeneous samples. In [19], both failure rates due to customer complaints in the first two years after delivery and based on a field study of many manufacturers in the midlife are reported. The rates are given relative to the total number of failures such as in our study, and show a wide range of occurrences, often with different classification criteria: for example, defect cell interconnects are around $10 \%$, j-box and cables around $10-20 \%$, glass failures 10-30\% depending on the plant age and means of detection. In [20], a physical model for degradation and reliability is validated on a large statistical data set, confirming that some studies do not distinguish for example corrosion damage from fatigue damage; however, more than $60 \%$ of returns is attributed in this case to cell-interconnection issues, considering modules that are aged less than 10 years. Kurtz et al. [21] discuss the degradation mechanisms depending on both technology and climate zones, as well as how they generally affect differently current and voltage giving average rates in terms of \%/year with no distinction based on the nature of defects. Finally, [22] reports a project report on PV plant risk assessment, finding that among the overall common failures, glass breakage, ethylene-vinyl acetate discoloration, and defective backsheets bear a higher level of economic impact on O\&M.

It is important to notice that UAV technology is too young to make available a large data set if compared for example with customer care data set. Even if the failure rates reported in this study are referred to ordinary or preventive maintenance in three of the five plants, some similarities with the literature can be found for the by-pass (j-box), cell, and hot-spot failures, while in our campaign we did not find high rate of glass breakage. Finally, a detailed comparison was not easy because of the different means of failure classification among the studies.

\section{CONCLUSION}

One of the most promising inspection methods for PV energy plants is the use of unmanned technology. Many modules installed in the recent past show a various range of defects which compromise their performance. This paper provides an overview 
of the most observed typical defects after a flight campaign made by ad-hoc designed light UAVs. Different kinds of sensors have been installed onboard and the results of this campaign have been discussed. Surely the efficiency of photovoltaic systems can be improved by appropriate use of O\&M instruments and benefit from innovative monitoring tools. The cost of unmanned system is still not negligible at the time, but their convenience is justifiable when a specific plant is subjected to serious failures. This paper has collected experimental data from multiple plants and the UAV-based monitoring concept has been validated in the field on a quite considerable number of heterogeneous plants in Italy. The evidence from these multiple inspections suggests that further lowering the operation cost will open up a wide market for O\&M more in general in the energy sector.

\section{REFERENCES}

[1] S. Djordjevic, D. Parlevliet, and P. Jennings, "Detectable faults on recently installed solar modules in Western Australia," Renew. Energy, vol. 67, pp. 215-221, Jul. 2014.

[2] E. L. Meyer and E. E. van Dyk, "Assessing the reliability and degradation of photovoltaic module performance parameters," IEEE Trans. Rel., vol. 53, no. 1, pp. 83-92, Mar. 2004.

[3] T. M. Walsh, Z. Xiong, Y. S. Khoo, A. A. Tay, and A. G. Aberle, "Singapore modules-Optimised PV modules for the tropics," Energy Procedia, vol. 15, pp. 388-395, 2012.

[4] P. Mints, "The commercialization of thin film technologies: Past, present and future," in Proc. 35th IEEE Photovolt. Spec. Conf., 2010, pp. 24002404.

[5] J. D. Bastidas-Rodríguez, E. Franco, G. Petrone, C. A. Ramos-Paja, and G. Spagnuolo, "Model-based degradation analysis of photovoltaic modules through series resistance estimation," IEEE Trans. Ind. Electron., vol. 62, no. 11, pp. 7256-7265, Nov. 2015.

[6] M. Aghaei, F. Grimaccia, C. Gonano, and S. Leva, "Innovative automated control system for PV fields inspection and remote control," IEEE Trans. Ind. Electron., vol. 62, no. 8, pp. 7287-7296, Aug. 2015.

[7] Y. Hu et al., "Online two-section PV array fault diagnosis with optimized voltage sensor locations," IEEE Trans. Ind. Electron., vol. 62, no. 11, pp. 7237-7246, Nov. 2015.

[8] D. Hausamann, W. Zirnig, G. Schreier, and P. Strobl, "Monitoring of gas pipelines-A civil UAV application," Aircr. Eng. Aerosp. Technol., vol. 77, pp. 352-360, 2005.

[9] A. Skoczek, "Long-term performance of photovoltaic modules," in Proc. 2nd Int. Conf. Sol. Photovolt. Investments, Feb. 2008, pp. 1-25.

[10] H. H. EI-Ghetany, G. E. Ahmad, H. M. S. Hussein, and M. A. Mohamad, "Long-term performance of photovoltaic modules at different tilt angles and orientations," in Proc. 37th Intersoc. Energy Convers. Eng. Conf., 2002, pp. 711-715.

[11] D. L. King, J. A. Kratochvil, M. A. Quintana, and T. J. McMahon, "Applications for infrared imaging equipment in photovoltaic cell, module, and system testing," in Proc. 28th IEEE Photovolt. Spec. Conf., 2000, pp. 1487-1490.

[12] ENAC Regulation, "Remotely piloted aerial vehicles," 2nd ed. Jul. 2015. [Online]. Available: https://www.enac.gov.it/repository/Content Management/information/N122671512/Reg_APR_Ed\%202_2.pdf

[13] H. Denio, "Aerial solar thermography and condition monitoring of photovoltaic systems," in Proc. 38th IEEE Photovolt. Spec. Conf., 2012, pp. 613-618.

[14] P. Bellezza Quater, F. Grimaccia, S. Leva, M. Mussetta, and M. Aghaei, "Light unmanned aerial vehicles (UAVs) for cooperative inspection of PV plants," IEEE J. Photovolt., vol. 4, no. 4, pp. 1107-1113, Jul. 2014.

[15] P. Manganiello, M. Balato, and M. Vitelli, "A survey on mismatching and aging of PV modules: The closed loop," IEEE Trans. Ind. Electron., vol. 62, no. 11, pp. 7276-7286, Nov. 2015.

[16] J.A. Tsanakas, G. Vannier, A. Plissonnier, D. L. Ha, and F. Barruel, "Fault diagnosis and classification of large-scale photovoltaic plants through aerial orthophoto thermal mapping," in Proc. 31st Eur. Photovolt. Sol. Energy Conf. Exhib., 2015, pp. 1783-1788.

[17] M. Aghaei, A. Dolara, S. Leva, and F. Grimaccia, "Image resolution and defects detection in PV inspection by unmanned technologies," in Proc. 2016 IEEE Power Energy Soc. Gen. Meeting, 2016, pp. 1-5.
[18] A. Dolara, G.C. Lazaroiu, S. Leva, G. Manzolini, and L. Votta, "Snail trails and cell microcrack impact on PV module maximum power and energy production," IEEE J. Photovolt., vol. 6, no. 5, pp. 1269-1277, Sep. 2016.

[19] M. Köntges et al., "Review on failures of photovoltaic modules," Int. Energy Agency Photovolt. Power Syst. Program., Rep. no. IEA-PVPS T13-01:2013, Mar. 2014.

[20] E. Hasselbrink et al., "Validation of the PVLife model using 3 million module-years of live site data," in Proc. IEEE 39th Photovolt. Spec. Conf., 2013, pp. 0007-0012. doi: 10.1109/PVSC.2013.6744087.

[21] S. R. Kurtz, J. H. Wohlgemuth, and D. C. Jordan, "Technology and climate trends in PV module degradation," in Proc. 27th Eur. Photovolt. Sol. Energy Conf. Exhib., Oct. 2012, pp. 3118-3124.

[22] D. Moser et al., "Identification of technical risks in the PV value chain and quantification of the economic impact," in Proc. 32nd Eur. Photovolt. Sol. Energy Conf. Exhib., 2016, pp. 1428-1434.

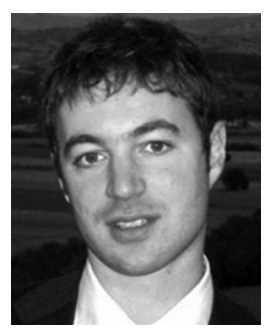

Francesco Grimaccia (M’07) received the M.S. and $\mathrm{Ph} . \mathrm{D}$. (cum laude) degrees in electrical engineering from the Politecnico di Milano, Milan, Italy, in 2003 and 2007, respectively.

Currently, he is an Associate Professor in the Energy Department, Politecnico di Milano. His main research interests include soft computing techniques development and application in different fields, such as wireless sensor networks, photovoltaic, and other energy harvesting devices.

Dr. Grimaccia is a member of CIS and SPIE and the Vice-President of AEIT-Milan section.

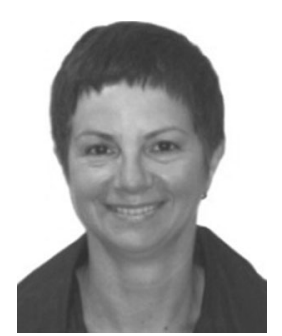

Sonia Leva (M'00-SM'13) received the M.S. and $\mathrm{Ph} . \mathrm{D}$. degrees in electrical engineering from the Politecnico di Milano, Milan, Italy, in 1997 and 2001, respectively.

She is a Full Professor of electrical engineering in the Department of Energy, Politecnico di Milano. Her research interests include EMC, power quality, and renewable energy analysis and modeling.

Dr. Leva is a member of the IEEE Working Group "Distributed Resources: Modeling \& Analysis" and the Task Force on "Modeling and Analysis of Electronically-Coupled Distributed Resources."

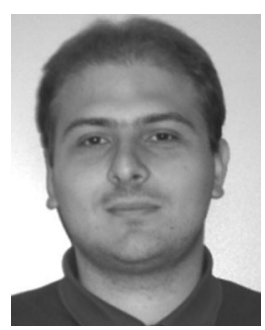

Alberto Dolara (S'09-M'13) received the M.S. and $\mathrm{Ph} . \mathrm{D}$. degrees in electrical engineering from the Politecnico di Milano, Milan, Italy, in 2005 and 2010, respectively.

$\mathrm{He}$ is an Assistant Professor in the Department of Energy, Politecnico di Milano. His research areas include traction systems, power quality, electromagnetic compatibility, and renewable sources.

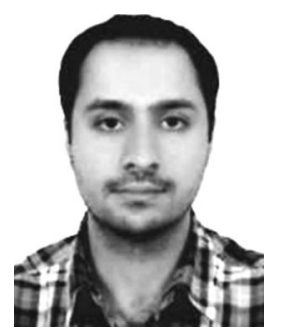

Mohammadreza Aghaei (S'14) received the B.S degree in electronics engineering from the Azad University, Tehran, Iran, in 2008, the M.S. degree in electrical engineering from the University Tenaga Nasional, Selangor, Malaysia, in 2013, and the Ph.D. degree in electrical engineering from the Politecnico di Milano, Milan, Italy, in 2016.

His research interests include power electronics, solar cells, photovoltaic systems, renewable sources, and UAVs. 